\title{
ORGANISMOS Y PROCESOS DE CERTIFICACIÓN DE CALIDAD DE LA EDUCACIÓN SUPERIOR PRIVADA EN MÉXICO
}

\section{Yazmín Cuevas $^{1}$}

\begin{abstract}
RESUMEN
En México los mecanismos oficiales que regulan la educación superior privada son muy flexibles, de suerte que existe una gran diversidad de instituciones de educación superior privadas (IESP): por un lado, instituciones que cumplen los elementos para ofrecer formación profesional; por otro, instituciones que apenas tienen los requisitos mínimos. Esto ha ocasionado una calidad académica desigual en las IESP, la cual se certifica (de manera voluntaria, es decir, no legalmente obligatoria) mediante tres organismos. En este artículo se analizan los procedimientos de los tres organismos, que se encargan de evaluar y certificar la calidad de las IESP. Se parte del supuesto de que estas certificaciones, por su carácter voluntario, tienen una limitada influencia en el mejoramiento de la calidad del sector superior privado, ya que pocas instituciones se someten a ellas.
\end{abstract}

Palabras clave: calidad, certificación, instituciones de educación superior privadas.

\section{ORGANISMS AND PROCESSES FOR THE QUALITY CERTIFICATION OF PRIVATE HIGHER EDUCATION IN MEXICO}

ABSTRACT

In Mexico, official mechanisms regulating private higher education are very flexible, thus, there is a wide variety of private higher education institutions: On the one hand, there are institutions that meet the requisites to provide professional training, on the other hand, there are institutions that barely meet the necessary requirements. This has caused an uneven academic quality among private higher education institutions. This quality is certified (on a voluntary basis, i.e. not legally binding) by three agencies. This article analyzes the procedures of the three agencies that are responsible for assessing and certifying the quality of private higher education institutions. It is assumed that, due to its voluntary nature, this evaluation process has limited influence on improving the quality of the private higher education system, since few institutions are subservient to it.

Keywords: quality; certification; private higher education institutions.

1 Programa de Licenciatura de Sociología de la Educación. Universidad Pedagógica Nacional. Ciudad de México, México. Contacto:yazco24@gmail.com 


\section{ORGANISMOS Y PROCESOS DE CERTIFICACIÓN DE CALIDAD DE LA EDUCACIÓN SUPERIOR PRIVADA EN MÉXICO}

\section{Introducción}

Durante los últimos treinta años el sector de educación superior privado en México ha tenido un crecimiento importante en relación con el sector público. Según datos de la Asociación Nacional de Universidades e Institutos de Educación Superior (ANUIES) el 32,2\% de los alumnos que cursan la licenciatura lo hacen en instituciones de educación superior privadas (IESP) (ANUIES, 2008). Este crecimiento se originó en la década de 1940, para atender a una formación profesional directiva y valórica que demandaban los grupos empresariales y católicos de la época. En 1943 se creó la Universidad Iberoamericana (UIA), en el mismo año el Instituto Tecnológico de Estudio Superiores de Monterrey (ITESM) y en 1946 el Instituto Tecnológico Autónomo de México (ITAM). Algunos autores denominan a estas primeras instituciones de élite, porque estaban destinadas a la formación de las clases altas (Silas, 2005; Mendoza, 2004; Levy, 1995).

Hasta finales de la década de 1970 la presencia de estas instituciones fue discreta. Así, en 1978 atendían al 12,4\% de la matrícula de licenciatura (Muñoz Izquierdo, 1981). A partir de 1980 comenzó la fundación de nuevas IESP, dirigidas a las clases media y media baja que no podían obtener un lugar en la educación superior pública. Al respecto, Silas (2005: 30) señala: "Estos impedimentos educativos corren de forma paralela con una de las premisas que más interesan a los gobiernos nacionales: la equidad. Estas instituciones [privadas] llevan oportunidades educativas de credencialización académica a las clases económicamente desfavorecidas (baja y media baja)". En efecto, a menudo las universidades, los centros o las escuelas de bajo costo son la única opción de la que disponen los estudiantes de sectores sociales débiles que no han cumplido los requisitos para ingresar al sector público. 
En este proceso de crecimiento la Secretaría de Educación Pública (SEP) no definió con precisión la regulación de las IESP, lo que trajo consigo normas muy flexibles y una calidad académica desigual. Consecuentemente existen, por un lado, instituciones que ofrecen licenciaturas en diferentes áreas de conocimiento (ciencias exactas, ciencias de la salud, administración y ciencias sociales, ciencias agropecuarias, humanidades y artes, por mencionar algunas), cuentan con una planta docente con una alta formación y de tiempo completo, tienen instalaciones adecuadas, desarrollan investigación y difunden la cultura; por otro lado, se puede encontrar IESP que sólo ofrecen licenciaturas en una o dos áreas de conocimiento (administración y ciencias sociales, humanidades y artes), carecen de profesores de tiempo completo, su infraestructura es insuficiente y no desarrollan investigación ni promueven la cultura.

En este artículo se analizan los procedimientos que ofrecen las tres organizaciones que se ocupan de evaluar y certificar la calidad de las instituciones que integran el sector privado de educación que son el Consejo para la Acreditación de la Educación Superior (COPAES), la ANUIES y la Federación de Instituciones Mexicanas Particulares de Educación Superior (FIMPES). Las tres tienen estándares coincidentes y procedimientos divergentes, y el propósito común de asegurar que las IESP cumplan con la calidad académica requerida para la educación superior. Sin embargo, sus certificaciones son voluntarias y opcionales. Por lo tanto, partimos de la premisa que al contar con tal carácter, las certificaciones son de limitada influencia al mejoramiento de la calidad académica del sector de educación superior privado, ya que pocas instituciones privadas se interesan en someterse a un proceso de esta naturaleza. Así, la mayoría de las IESP que cuentan con varias o alguna certificación de calidad son de perfil medio o alto.

\section{La expansión de las IESP desde 1980 y su diversidad}

Desde principios de la década de 1980 las instituciones de élite comenzaron a incrementar su matrícula de atención y abrieron más planteles. Paralelamente comenzaron a crecer otras alternativas de estudios profesionales denominadas de atención a la demanda que poco a poco adquirieron mayor presencia (Leonardo, 1983). Estas 
instituciones surgieron a partir de 1970 como educación comercial (escuelas técnicas, secretariado y escuelas normales) que aunque no ofrecían educación superior trataban de ser una opción atractiva de educación técnica; en la década de los ochenta algunos de estos centros se transformaron en instituciones de educación superior. Para Leonardo (1983), el rápido crecimiento de las instituciones privadas a partir de los años ochenta se debió a que los sectores conservadores de la sociedad veían en las universidades públicas una gran politización, se percibía que la formación que se ofrecía era de mala calidad y no garantizaba la obtención de un empleo. Por otro lado, a pesar de la masificación de las universidades públicas ya no se podía garantizar el ingreso de más alumnos. Como consecuencia de las crisis económicas de los años ochenta (1982 y 1987), la inversión del gasto para la educación superior disminuyó. En 1980 el gasto para este nivel era del 0,6\% del producto interno bruto (PIB) y para 1990 fue del 0,4\% (Casanueva, 2005), esto indica que hubo una desaceleración en la inversión de la educación superior pública.

Tabla 1. Crecimiento del sector de educación superior privado (técnico superior universitario, licenciatura universitaria y tecnológica y licenciatura normal)

\begin{tabular}{|c|c|c|c|c|c|}
\hline Año & Público & $\%$ & Privado & $\%$ & Total \\
\hline 1980 & 632307 & 86,5 & 98840 & 13,5 & 731147 \\
\hline 1985 & 810391 & 84,2 & 151077 & 15,8 & 961468 \\
\hline 1990 & 890372 & 82,6 & 187819 & 17,4 & 1078191 \\
\hline 1995 & 943245 & 77,5 & 274186 & 22,5 & 1217431 \\
\hline 2000 & 1119170 & 70,6 & 466238 & 29,4 & 1585408 \\
\hline 2005 & 1517521 & 67,9 & 716485 & 32,1 & 2234006 \\
\hline 2008 & 1610663 & 67,8 & 788422 & 32,2 & 2449085 \\
\hline
\end{tabular}

Elaboración propia sobre la base de los anuarios estadísticos de la ANUIES (2008, 2006, 2003, 1996, 1990, 1986, 1981).

Aunque en los últimos 28 años el sector público ha incrementado la matrícula en el nivel superior, porcentualmente su cobertura ha ido decreciendo en relación con el sector privado, en 1980 atendía al $86,5 \%$ y para 2008 el porcentaje disminuyó a $67,8 \%$, es decir, casi un $20 \%$. Mientras el sector privado se ha expandido significativamente (tanto en matrícula, como porcentualmente), en 1980 absorbía el 13,5\% de la matrícula de educación superior, en 2008 duplicó su porcentaje $(32,2 \%)$. Así, este sector privado ha crecido en veintiocho 
años un $23 \%$. Estas cifras evidentemente denotan que la educación superior privada puede continuar incrementando su oferta hasta igualar y superar la absorción de la demanda de la educación superior pública. La expansión se debe principalmente a cuatro factores:

- El primero, desde mediados del siglo XX México estableció políticas para que se universalizara la educación básica, lo que ha generado un importante número de aspirantes a cursar el nivel de educación media superior y superior. No obstante, el Estado no ha podido satisfacer la demanda de educación superior pública.

- El segundo, las IESP reciben a estudiantes que la educación pública no puede atender. Una de las medidas para satisfacer la demanda en la educación superior son las universidades, los centros o las escuelas de bajo costo a los que pueden acceder estudiantes de sectores sociales débiles que no han cumplido los requisitos para ingresar al sector público. En palabras de Vega-Tanto (2009: 38): "La demanda crece y no tiene cabida en las instituciones públicas, de manera que las particulares además de atraer a sus propias clientelas, cumplen la función de atender a una buena parte de los miles de jóvenes rechazados de todo el país y de aquello que forman parte del rezago no atendido".

- El tercer factor, las IESP ofrecen programas académicos en las áreas de ciencias sociales y administrativas, las ingenierías y tecnología porque tienen un bajo costo para la institución y paralelamente son programas muy atractivos para los aspirantes a cursar educación superior (Acosta, 2005). Según datos de ANUIES (2008) en el sector privado las áreas que concentran la mayor matrícula son: ciencias sociales y administrativas con 38,3\% e ingeniería y tecnología con $38,3 \%$, las que en conjunto concentran el 76,6\% de los estudiantes del sector privado.

- El cuarto, la incorporación de las mujeres al sector superior, para Acosta (2005:30) "la feminización de la matrícula es un fenómeno generalizado en el sistema de educación superior de México y América Latina". Lo anterior se corrobora con cifras, ya que en 2008 la matrícula de estudiantes mujeres del nivel de educación superior ocupaba el 48,8\%, y específicamente en el sector privado ascendía al 55\% (ANUIES, 2008). 
El crecimiento del sector superior privado trajo consigo la aparición de una diversidad de instituciones (Acosta, 2005), debido a que existen universidades, institutos, centros que pueden ser religiosos o confesionales, de élite, o bien escuelas, universidades, centros que están dirigidos a brindar atención a la demanda y que difícilmente se conoce la calidad educativa con la que operan. Dada esta heterogeneidad, la preocupación de los expertos en educación superior, es que no se puede colocar en un mismo nivel a todas estas instituciones ya que existen diferencias importantes entre cada una de ellas, desde la orientación ideológica hasta el tipo de instalaciones que tienen. Para Silas (2005), por la calidad que ofrecen las IESP, se pueden clasificar en:

a) Perfil alto. Cumplen con todos los requisitos para otorgar educación superior como instalaciones adecuadas, planta de profesores con posgrado y contratación de tiempo completo, desarrollan investigación. Acceden a ellas los grupos de estudiantes con mayor afluencia económica. Son de élite y según Mendoza "clasificamos en este sector a las instituciones cuya clientela es reclutada mayoritariamente en las clases altas de la sociedad" (2004: 332). Surgieron como oposición a la tradición estatista que dominaba en las universidades públicas y son producto de las cúpulas empresariales y la clase política.

b) Perfil medio. Realizan mejoras para cumplir con los requisitos de calidad académica ampliando instalaciones, contratando profesores con posgrado, comienzan a realizar investigación. Para Mendoza (2004) son instituciones que surgieron para atender a la demanda, pero que al paso del tiempo se han consolidado, ofrecen una amplia gama de carreras, infraestructura, organización eficiente, cuentan con una matrícula mayor de 3.000 alumnos y son una opción para sectores medios de la población.

c) Perfil bajo. No reúnen los criterios de calidad necesarios, tienen instalaciones deficientes, sus profesores son contratados a tiempo parcial, no realizan investigación. Para Mendoza son "aquellas que reclutan a sus estudiantes entre los sectores medios de la población impedidos de pagar colegiaturas de las instituciones de élite o bien rechazados de la universidad pública, cuyo ingreso ha sido muy competido" (2004: 335). Son instituciones pequeñas, con una 
matrícula y oferta reducida, generalmente de corte administrativo y comercial, sin una infraestructura adecuada.

Con esta heterogeneidad de IESP salta a la vista los diferentes rangos de calidad, es decir, hay una desigualdad entre los estudiantes de este sector, dependiendo de sus posibilidades económicas pueden acceder a instituciones de alto nivel académico o bien incorporarse a instituciones de bajo perfil. Así, al hablar de educación superior privada no se alude necesariamente a instituciones que cumplen con los criterios de calidad académica, sino a diferentes opciones que de acuerdo con el sector socioeconómico al que están dirigidas ofrecen distintos niveles de calidad.

\section{Autorización para otorgar educación superior}

La diversidad institucional del sector de educación superior privado, ha sido consecuencia de la normatividad que la regula. Las IESP en México para ofrecer formación profesional necesitan de un reconocimiento oficial, que puede solicitarse por dos vías: tramitando un Reconocimiento de Validez Oficial de Estudios (RVOE) en la SEP o la incorporación a alguna universidad pública o autónoma.

La primera forma implica que para cada programa académico (licenciaturas o posgrados) se debe solicitar un RVOE. En el acuerdo 279 (SEP, 2002) se indican las condiciones que tienen que acatar las instituciones para solicitar dicho reconocimiento, entre las que destacan las instalaciones (espacios comunes, laboratorios, mobiliario, biblioteca), la formación y contratación de los docentes y los planes y programas de estudios. Tras una visita, por parte de la Secretaría, a la institución interesada y la revisión de la documentación se otorga el RVOE. Anualmente la SEP realiza dos supervisiones a las instituciones privadas para corroborar que se cumple con los requisitos indispensables para ofrecer educación superior; no obstante, Casanueva (2005) indica que debido al gran número de instituciones privadas en todo México es muy difícil que esto se lleve a cabo.

Una de las preocupaciones de los expertos es que, según la oficina (federal o estatal), existe una aplicación distinta de los 
criterios para otorgar el RVOE (Vega-Tanto, 2009; Olivier, 2005). En el "RVOE pueden propiciarse movimientos académicos irregulares, no necesariamente ilícitos desde el punto de la Ley General de Educación. La contraparte es que la autoridad estatal puede tener procedimientos discrecionales en cuanto a la fijación de condiciones que deben cumplir los establecimientos de esta categoría" (Olivier, 2005: 140). Así, este trámite no es una garantía de que los estudios que brindan las IESP cumplan con los requisitos mínimos de calidad académica.

Mediante la segunda opción, las IESP deben seguir los programas de estudios de las instituciones receptoras, tener profesores con un perfil determinado e instalaciones adecuadas (como biblioteca, laboratorios, talleres). La institución que otorga la incorporación establece los criterios, los tiempos y las formas de supervisión (Levy, 1995). Al parecer no es fácil conseguir una autorización de este tipo y es más sencillo tramitar un RVOE, por lo que la gran mayoría opta por lo último (Rodríguez, 2005; Olivier, 2005).

Una vez que las IESP cumplen con cualquiera de estos dos trámites pueden impartir educación superior; pero esto no avala la calidad de estas. Para este propósito la certificación ${ }^{2}$, que permite a las instituciones privadas la oportunidad de solicitar la evaluación a una agencia acreditadora reconocida por el COPAES, pertenecer a la ANUIES mediante la evaluación diagnóstica de la calidad académica de la institución, o bien someterse a un autoestudio de incorporación a la FIMPES.

\section{La certificación del COPAES: criterios, indicadores y parámetros generales}

El COPAES es "una asociación civil cuya misión es contribuir al aseguramiento de la calidad de los programas académicos que se ofrecen en las instituciones públicas y particulares de México, mediante el reconocimiento formal de las organizaciones de acreditación que

2 Se entiende por certificación al reconocimiento que otorga alguna instancia pública o privada, sobre la calidad académica de determinada institución superior. Para ello, la institución debe someterse a un proceso de evaluación. Véase Villaseñor (2004). 
demuestren la idoneidad, calidad y confiabilidad de sus procesos y resultados, y que desarrollen sus funciones y procesos con base en los Lineamientos y en el Marco General para los Procesos de Acreditación de Programas Académicos de Nivel Superior, establecidos por el mismo Consejo"(COPAES, 2010a). Este Consejo cuenta con 29 agencias acreditadoras reconocidas y cuatro en proceso de reconocimiento. Sin embargo -debido a que las agencias son de carácter particular y con fines de lucro-, uno de los debates en la opinión pública que se ha presentado es que el proceso de certificación de la calidad académica de los programas puede ser controvertido.

Cada agencia define su metodología de evaluación y certificación, la cual necesita estar apegada a los lineamientos y al marco general dictados por el COPAES. Así, la metodología debe contemplar (COPAES, 2010b):

- Categorías, factores o áreas: el personal académico adscrito al programa, el plan de estudios, los métodos e instrumentos para la evaluación del aprendizaje, los servicios institucionales para el aprendizaje de los estudiantes, los alumnos, la infraestructura y equipamiento de apoyo al programa, las líneas y actividades de investigación, vinculación institucional, conducción académicoadministrativa, y los procesos de planeación y evaluación, gestión y financiamiento.

- Criterios: referentes de valoración establecidos previamente.

- Indicadores para cada categoría, factor o áreas: "Se precisará la expresión cuantitativa y cualitativa del valor de dos o más propiedades del fenómeno" (COPAES, 2010b: 8).

- Parámetros, estándares o referentes de evaluación: los valores que se deben cumplir y que se manifiestan mediante indicadores.

Los propósitos de la acreditación del COPAES son reconocer de manera pública la calidad de los programas académicos, fomentar una cultura de mejora continua, y que las instituciones alcancen parámetros de calidad nacional e internacional (COPAES, 2010b). Para participar en este proceso se realiza la solicitud por programa académico, es decir, por cada licenciatura, especialización, maestría o doctorado. El proceso consta de tres fases: 
- En la primera fase, la institución realiza una autoevaluación cuantitativa y cualitativa acerca del programa, que contemple las actividades formativas, la organización, las instalaciones, el funcionamiento y se elabora un reporte que se entrega al organismo acreditador. En esta autoevaluación es necesario que se involucre a la comunidad académica del programa.

- En la segunda, la agencia certificadora realiza una evaluación al programa.

- En la tercera, la agencia emite una valoración que dará como resultado el dictamen final: la acreditación del programa, o bien la no acreditación.

De acuerdo con datos de la Subsecretaría de Educación Superior (2010) hasta el 31 de marzo de 2010, sólo 537 programas educativos (licenciaturas, especialidades, maestrías, doctorados) de instituciones del sector privado contaban con la acreditación del COPAES. Cabe aclarar que generalmente las IESP certificadas poseen más de un programa en el COPAES, por ejemplo, el ITSEM tiene 200 programas, la Universidad del Valle de México, 54; la UIA, 27; la Universidad Lasalle (ULSA), 25; la Universidad Anáhuac del Sur, 23; el Instituto Tecnológico y de Estudios Superiores de Occidente (ITESO), 21; la Universidad Panamericana, 19 (Subsecretaría de Educación Superior, 2010). También hay que señalar que casi todas estas instituciones son catalogadas como de alto perfil, que imparten educación superior cobrando altas colegiaturas y dirigidas a los grupos más favorecidos económicamente.

\section{ANUIES: diagnóstico evaluativo y comité de expertos}

La ANUIES es una organización no gubernamental que agrupa a instituciones mexicanas públicas y privadas (ANUIES, 2010). Desarrolla investigaciones sobre la educación superior, forma a los profesores de este nivel, genera innovaciones educativas para el mejoramiento de la educación superior, publica la Revista de la Educación Superior, promueve la calidad de las instituciones que agrupa, realiza estudios estratégicos que permitan la toma de decisiones para la educación superior, apoya el intercambio interinstitucional, establece convenios 
con instituciones extranjeras y con el sector productivo del país (ANUIES, 2010).

Es evidente que la finalidad de la ANUIES no es únicamente certificar a las IESP; no obstante tiene una amplia experiencia al respecto, ya que ha participado en la fundación de organizaciones que tienen el propósito de mejorar la calidad de las instituciones de educación superior, como la Comisión Nacional de Evaluación, los Comités Interinstitucionales para la Evaluación de la Educación Superior, el Centro Nacional de Evaluación para la Educación Superior.

Para solicitar el ingreso es necesario que la institución pública o privada ofrezca docencia, desarrolle investigación, realice difusión de la cultura, tenga matriculados a por lo menos mil alumnos en licenciatura o cien alumnos en posgrado, cuente con egresados de licenciatura con el título o egresados de posgrado que hayan obtenido el grado. Es decir, desde el momento que cualquier institución de educación superior se interesa por pertenecer a la ANUIES debe contar con condiciones indispensables para ser considerada al proceso de evaluación diagnóstica.

Las instituciones interesadas, en el caso que nos ocupa, a saber, las privadas, deben realizar una petición formal de ingreso al Consejo Regional de la ANUIES, el cual indicará los documentos y requisitos por reunir. Así, el Consejo Regional designa a un comité de expertos para que analicen los documentos presentados y realicen una visita a la institución. Este comité elabora un diagnóstico evaluativo sobre la calidad de la institución mediante criterios e indicadores emitidos por la ANUIES que arrojarán datos importantes sobre el estado y calidad de la institución. Posteriormente, el Consejo Regional, basado en un reporte elaborado por el comité de expertos, presenta un informe final al Consejo General de la asociación, que delibera el ingreso de la institución interesada (Fresán y Taborga, 2002).

El diagnóstico evaluativo se compone de 61 indicadores, de carácter cuantitativo, que analizan diez áreas de las instituciones de educación superior (Fresán y Taborga, 2002): 
- Naturaleza y misión de la institución. Se identifica que la institución tenga una misión afín a las actividades académicas que realiza y que sus resultados puedan ser evaluados. En esta área especialmente se pone atención a que la institución cuente con antigüedad mínima de diez años, una matrícula determinada, documentos institucionales donde se exprese su misión.

- Normatividad institucional. Se verifica que la institución cuente con normas que regulen sus actividades, lo que implica tener un reglamento para los profesores (que especifique cuestiones relacionadas con el ingreso, permanencia y promoción) y otro para estudiantes (que regule ingreso, promoción y egreso), así como normas para la participación de profesores y estudiantes en los órganos de la dirección.

- Planeación. Se indaga sobre el proceso de planeación para alcanzar los niveles de calidad deseados, se examinan los mecanismos que la institución sigue como el establecimiento de políticas de desarrollo institucional, la elaboración y seguimiento de la planeación institucional, y los procesos de dicha evaluación de la planeación.

- Infraestructura. Se constata que cuente con las instalaciones y servicios necesarios para la formación profesional, la investigación y la difusión cultural, como recursos bibliográficos, aulas, oficinas, talleres, laboratorios y recursos informáticos.

- Planes de estudios. Se analiza que la institución ofrezca planes de estudios actuales y pertinentes a las necesidades de la sociedad. Se presta atención a los procedimientos para generar y evaluar los planes y programas de estudio, la congruencia entre estos y el proyecto educativo, la compatibilidad de la titulación y la graduación con los mismos.

- Evaluación. Se verifica que se cuente con procedimientos claros y precisos para la valoración de los alumnos y los docentes, además de efectuar estudios de seguimiento de egresados.

- Profesores. Se indaga sobre la pertinencia de la planta de profesores para la atención de los programas educativos. En este caso se pone especial atención en el nivel académico de los profesores (licenciatura, maestría, doctorado), número de profesores de tiempo completo y de tiempo parcial y la participación de 
los profesores en proyectos de investigación, programas de divulgación científica y tecnológica.

- Estudiantes. Se identifica que se cuente con un sistema de ingreso y seguimiento de estudiantes para lo cual deben existir criterios de ingreso, programas de atención, investigaciones sobre eficiencia terminal, rezago y deserción.

- Recursos financieros. Se comprueba que se tengan normas y procedimientos para la gestión de los recursos monetarios. La institución debe tener una normatividad financiera, procedimientos para la elaboración y evaluación del presupuesto anual, y normas con respecto a los balances financieros.

- Difusión cultural. Se conoce la existencia de programas y proyectos para la difusión y extensión cultural. Esto implica que se cuente con revistas científicas con reconocimiento del Consejo Mexicano de Ciencia y Tecnología (CONACyT), colecciones de libros que sean arbitrados, salas de exposiciones, teatros, programas de educación continua.

Se debe precisar que algunas de las instituciones que son aceptadas como miembros por la ANUIES no cumplen con algunos de los indicadores establecidos. Así, el Consejo Nacional de la asociación diseña programas que permitan que se alcancen los criterios faltantes en un plazo de cinco años (Fresán y Taborga, 2002). Hasta octubre de 2010, pertenecían a la ANUIES 152 universidades de instituciones de educación superior, de las cuales 21 eran de carácter privado, es decir, solo 1,3\% (ANUIES, 2008). Destacan el ITSEM, el ITAM, la UIA, la ULSA, el ITESO. Al igual que con el COPAES, se repite la tendencia, solo las instituciones de alto perfil son las que cumplen los requisitos de certificación.

\section{FIMPES: autoestudio}

Otra manera de certificar la calidad del sector privado es mediante la FIMPES, que es una asociación civil que conformaron las IESP en 1981 con el propósito de establecer una colaboración entre ellas. En sus inicios el ingreso a la FIMPES era libre, pero desde 1996 se estableció un proceso de certificación al que deben someterse las instituciones 
privadas que deseen incorporarse. Esta certificación consta de cuatro fases (FIMPES, 2009):

- En la primera fase la IESP solicita por escrito la incorporación; en seguida, algunos de los miembros de la FIMPES hacen una visita para hacer una valoración y se emite la aceptación de la IESP como miembro aspirante. Con todo, hay que advertir que en la documentación oficial de la FIMPES no se encontraron los criterios que se siguen para aceptar o rechazar a una institución como miembro aspirante.

- La segunda fase implica que la institución de educación emprenda un autoestudio con duración de dos años, para lo cual la FIMPES asigna a un representante que apoya el proceso (que no participará en la etapa de verificación). Para realizar este autoestudio la institución conforma comités que se encargarán de las áreas por evaluar y elabora un manual interno para el autoestudio (que contempla el propósito, la organización de personal y recursos, la asignación y responsabilidad de comités, los programas de actividades, la metodología, los formatos de redacción, las políticas, la normas y los procedimientos del autoestudio y la bibliografía). Sobre la base de la metodología establecida en el manual se recopila la información y se redacta el informe del autoestudio que debe ir avalado por el representante de FIMPES. Todos los miembros del plantel educativo (funcionarios, académicos y administrativos) se deben involucrar en la evaluación de cada una de las áreas que integran la institución y, con ello, se pueden ir realizando ajustes para el mejoramiento de la calidad académica. Al final se entrega un reporte del resultado del autoestudio.

- La tercera etapa es de verificación, que consiste en que un equipo de miembros de la Federación confronte la información presentada en el autoestudio con la realidad institucional. El equipo de verificación elabora un informe final sobre el estado de la institución a FIMPES.

- Para la cuarta etapa, la Secretaría General de la FIMPES emite un estatus de la certificación que puede ser:

a) Liso y llano (se le conoce de excelencia académica y es reconocido ante la SEP, para realizar trámites del RVOE).

b) Aprobado sin observaciones. 
c) Aprobado con recomendaciones, las cuales deben ser realizadas en un periodo determinado.

d) Aprobado con observaciones, que necesitan ser cumplidas para conservar la acreditación.

Los criterios en los cuales se apoya el autoestudio se denominan "debes" y son 180 indicadores de calidad que contemplan la filosofía institucional, la planeación institucional, la estructura organizacional, los programas educativos y la investigación, la calidad del personal académico, los programas de apoyo extraescolar dirigidos a los estudiantes, la gestión escolar, las condiciones laborales del personal administrativo, la infraestructura (biblioteca, talleres, laboratorios, sistema de cómputo) y la educación a distancia en caso de que la institución ofrezca esa modalidad educativa (FIMPES, 2010) ${ }^{3}$. Cada cinco años las IESP afiliadas a FIMPES inician nuevamente el proceso de autoevaluación (con duración de dos años) con el fin de refrendar su acreditación.

Hasta octubre de 2010 la FIMPES contaba con 67 instituciones acreditadas, de las cuales 39 tenían el estatus de lisa y llana, 22 sin observaciones; cinco con recomendaciones y una con condiciones. Además, 42 IESP estaban realizando su autoestudio para la acreditación. De acuerdo con Vega y Tanto, solo 10\% de las IESP cuenta con la acreditación de la FIMPES. Entre las instituciones que tienen la acreditación lisa y llana destacan el ITAM, el ITESO, la UIA, la ULSA, la Universidad Anáhuac. Nuevamente, como en la certificación del COPAES y la ANUIES, se trata de las mismas instituciones, aquellas que tienen un perfil alto que atiende a las clases con mayores recursos económicos.

\section{Análisis comparativo de los tres organismos certificadores: metodología y áreas para evaluar}

Hasta el momento se ha explicado de manera general los procedimientos de cada uno de los organismos que se encargan de certificar a las

3 De acuerdo con Ríos (2001) la FIMPES para el diseño de sus debes se apoyó en el modelo de acreditación de Southern Association of Colleges and Schools, agencia acreditadora de los Estados Unidos de Norteamérica. 
IESP. Este apartado se centrará en realizar una comparación en la metodología que siguen y las áreas que se evalúa.

Metodología. Es el conjunto de procedimientos y el método que adopta el organismo certificador en el proceso de la evaluación a la institución de educación superior. Así, se comparó el carácter de la certificación (obligatorio o voluntario), el método de evaluación, el procedimiento y el número de indicadores (ver tabla 2).

Tabla 2. Organismos de certificación de calidad dirigidos a IESP

\begin{tabular}{|l|l|l|l|}
\hline & COPAES & ANUIES & FIMPES \\
\hline $\begin{array}{l}\text { Carácter de la } \\
\text { certificación }\end{array}$ & Voluntario & Voluntario & Voluntario \\
\hline Método & $\begin{array}{l}\text { Autoevaluación y } \\
\text { evaluación. }\end{array}$ & $\begin{array}{l}\text { Evaluación diagnóstica. } \\
\text { Procedimiento }\end{array}$ & $\begin{array}{l}\text { Autoevaluación en } \\
\text { donde participan todos } \\
\text { los miembitución diseña la la } \\
\text { institución. } \\
\text { metodología). }\end{array}$ \\
\hline $\begin{array}{l}\text { Posterior, una evaluación } \\
\text { al programa por parte de } \\
\text { la agencia certificadora. }\end{array}$ & $\begin{array}{l}\text { Diagnóstico evaluativo } \\
\text { realizado por un comité } \\
\text { de expertos designados } \\
\text { de la ANUIES. }\end{array}$ & $\begin{array}{l}\text { Autoestudio en el } \\
\text { que participan todos } \\
\text { los miembros de la } \\
\text { institución. } \\
\text { Posterior un comité de } \\
\text { la federación verifica el } \\
\text { reporte final con una } \\
\text { visita a la institución. }\end{array}$ \\
\hline Indicadores & $\begin{array}{l}\text { Solo se indican las áreas } \\
\text { del programa que se } \\
\text { deben evaluar, cada } \\
\text { agencia establece sus } \\
\text { indicadores. }\end{array}$ & 57 indicadores. & 180 indicadores. \\
\hline
\end{tabular}

En el análisis se encontró que cada organismo sigue una metodología distinta. El COPAES evalúa, como se ha señalado, por programa académico. Su método consta de dos fases, en la primera la institución que se va a certificar sigue un proceso de autoevaluación de sus actividades, organización, procesos, metas y resultados (COPAES, 2010b). En la segunda, el organismo acreditador efectúa una evaluación, cabe destacar que no se tienen datos sobre esta fase, ya que cada agencia certificadora (perteneciente al Consejo) establece su método y sus indicadores de calidad. La ANUIES sigue un método distinto, es un diagnóstico evaluativo, mediante 57 indicadores se determina la situación de la calidad de la institución de educación superior (Fresan y Taborga, 2002). Sin embargo, 
para solicitar este diagnóstico la institución que desea evaluarse desde el principio tiene que cumplir con condiciones necesarias (docencia, investigación, difusión cultural, número determinado de alumnos). El objetivo de la asociación es apoyar a las instituciones que no alcancen todos los criterios del diagnóstico evaluativo, para establecer acciones y lograr la calidad académica. La FIMPES tiene un procedimiento distinto al de los otros organismos. En principio a la evaluación se le denomina autoestudio, es decir, realizada por los miembros de la institución que a la par van haciendo reformas para fomentar la calidad. El proceso es largo, dos años, y la metodología es definida por la institución que desea certificarse. Finalmente, un comité de la federación corrobora que se cumplan 180 indicadores de calidad.

Así, las metodologías de evaluación son distintas, cada una de ellas está determinada por los propósitos de cada organismo certificador. Para el COPAES su objetivo es reconocer la calidad académica de los programas educativos (licenciaturas, maestrías, doctorados). La ANUIES promueve la calidad académica de la educación superior, como consecuencia las instituciones que demuestran los criterios indispensables para solicitar el ingreso siguen una metodología que les ayudará a diagnosticar sus fortalezas y debilidades con el fin de mejorar la calidad. La FIMPES agrupa y propicia la colaboración entre instituciones privadas, es por eso que se sigue una autoevaluación que permitirá a la IESP ajustarse a los criterios de calidad.

Tanto en el método del COPAES y la FIMPES se logra identificar que es el personal de la institución el que realiza la evaluación, lo cual se conoce como autoevaluación. Este proceso requiere de un acompañamiento por parte de un experto para orientar y, sobre todo, para constatar que la IESP está realizando cambios con el fin de mejorar su calidad académica. En esta dirección, se considera que el proceso del COPAES es más completo que el de la FIMPES, porque una vez finalizada la autoevaluación se emprende una evaluación a cargo de la agencia certificadora. Con esta se garantiza que la IESP ha cumplido con los estándares de calidad. Por su parte, el procedimiento de la FIMPES tiene un debilidad importante, una vez que la IESP concluye 
su autoestudio los representantes de la federación efectúan una visita para corroborar que se cumplió con modificaciones y mejoras a la institución privada; con estos datos se elabora un reporte final. Es decir, solo se verifican los cambios, pero no se conoce a profundidad cómo la IESP desarrolló su autoevaluación. Esto se puede prestar a que la IESP realice algunas modificaciones para la visita de verificación y que no haya transformaciones mayores que repercutan en la calidad académica de la institución. Sin duda el proceso de evaluación más exhaustivo es el de la ANUIES, dado que es un comité de expertos es el que realiza la evaluación que arrojará un diagnóstico sobre el estado de calidad de la IESP. Se destaca que, desde el inicio la ANUIES se involucra en la evaluación a la institución; con ello se puede tener una visión completa de la calidad de la institución de educación superior.

Áreas para evaluar. Cada organismo presenta las áreas de las instituciones que deben ser evaluadas. El COPAES establece doce, la ANUIES diez y la FIMPES once. Los tres organismos coinciden en siete áreas (ver tabla 3), que son: planeación, normatividad, recursos financieros, infraestructura, planes de estudio, planta docente y estudiantes.

Con el análisis de estas áreas para evaluar, particularmente la del personal académico, se identificó que los tres organismos consideran aspectos distintos. La ANUIES presta mayor atención a que los profesores desarrollen investigación, la FIMPES a la actualización docente y el COPAES a la distribución de cargas académicas. Existen áreas en la federación como la de planes de estudio que comprenden muchas actividades como programas educativos, evaluación de aprendizajes, desarrollo de la investigación y la difusión cultural. Así, aunque cada organismo tiene áreas de evaluación similares, éstas pueden integrarse por muchas actividades. Por ejemplo, para ANUIES la difusión cultural es un área para diagnosticar y para la FIMPES es un aspecto que conforma al área de programas educativos. 
Tabla 3. Áreas de evaluación por organismo

\begin{tabular}{|c|c|c|c|}
\hline Áreas para evaluar & COPAES & ANUIES & FIMPES \\
\hline Filosofía institucional & & $\begin{array}{l}\text { Naturaleza y misión de } \\
\text { la institución. }\end{array}$ & Filosofía institucional. \\
\hline Planeación & $\begin{array}{l}\text { Proceso de evaluación y } \\
\text { planeación. }\end{array}$ & Planeación. & $\begin{array}{l}\text { Planeación y } \\
\text { efectividad. }\end{array}$ \\
\hline Normatividad & $\begin{array}{l}\text { Normatividad } \\
\text { institucional que } \\
\text { regule la operación del } \\
\text { programa. }\end{array}$ & $\begin{array}{l}\text { Normatividad } \\
\text { institucional. }\end{array}$ & $\begin{array}{l}\text { Normatividad, } \\
\text { gobierno y } \\
\text { administración. }\end{array}$ \\
\hline Recursos financieros & $\begin{array}{l}\text { Gestión administrativa } \\
\text { y actividad financiera. }\end{array}$ & Recursos financieros. & Recursos financieros. \\
\hline Gestión & $\begin{array}{l}\text { Conducción académico- } \\
\text { administrativa. }\end{array}$ & & \\
\hline Infraestructura & $\begin{array}{l}\text { Infraestructura y } \\
\text { equipamiento de apoyo } \\
\text { al programa. }\end{array}$ & Infraestructura. & Recursos físicos. \\
\hline Apoyos académicos & & & Apoyos académicos. \\
\hline Plan de estudios & Curriculum. & Planes de estudios. & $\begin{array}{l}\text { Programas educativos } \\
\text { y la investigación. }\end{array}$ \\
\hline Planta docente & $\begin{array}{l}\text { Personal académico } \\
\text { adscrito al programa. }\end{array}$ & Profesores. & Personal académico. \\
\hline Estudiantes & Alumnos. & Estudiantes. & Estudiantes. \\
\hline $\begin{array}{l}\text { Servicios de apoyo a } \\
\text { estudiantes }\end{array}$ & $\begin{array}{l}\text { Servicios institucionales } \\
\text { para el aprendizaje. }\end{array}$ & & \\
\hline Evaluación & $\begin{array}{l}\text { Métodos e } \\
\text { instrumentos para } \\
\text { evaluar el aprendizaje. }\end{array}$ & Evaluación. & \\
\hline & Vinculación. & & \\
\hline Investigación & Investigación. & & \\
\hline Difusión cultural & & Difusión cultural. & \\
\hline $\begin{array}{l}\text { Personal } \\
\text { administrativo }\end{array}$ & & & $\begin{array}{l}\text { Personal } \\
\text { administrativo. }\end{array}$ \\
\hline Educación a distancia & & & $\begin{array}{l}\text { Educación a distancia } \\
\text { (si se ofrece la } \\
\text { modalidad). }\end{array}$ \\
\hline
\end{tabular}

Se encontró que los tres procesos de evaluación de los organismos responden a intenciones muy concretas. Para el COPAES, al ser una certificación por programa, se presta mayor atención a áreas que evidencian la operación como plan de estudios, servicios de apoyo al estudiante y la investigación. La ANUIES fija sus áreas en relación con un sentido amplio de la educación superior que tiene como propósito la formación profesional, el desarrollo de la investigación y la difusión cultural. La FIMPES desde sus inicios ha estado interesada en que las IESP 
cuenten con una organización básica para ofrecer formación profesional, con lo cual en su autoestudio el mayor peso se le da a la planeación, la infraestructura, los recursos académicos, los programas educativos y los estudiantes. No necesariamente las instituciones que pertenecen a FIMPES deben desarrollar investigación y difundir la cultura.

Los tres organismos parten del principio que las IESP aseguran su calidad académica al cubrir la filosofía institucional, la planeación estratégica, el personal académico con una alta formación (posgrado), la atención a alumnos, el desarrollo de investigación y difusión cultural. Sin embargo, no hay estudios que se hayan encargado hasta el momento de analizar la pertinencia de cada una de las metodologías de estos organismos y sus respectivas áreas de evaluación. Probablemente por la experiencia en el desarrollo de políticas y programas de evaluación, es la ANUIES la que ofrece una transparencia en su proceso de evaluación diagnóstica, ya que existe una información muy extensa y precisa (Fresán y Taborga, 2002). La debilidad que presentan tanto las áreas para evaluar así como sus criterios, del COPAES y la FIMPES, es que existe poca información al respecto, sobre todo, no se encontró para la elaboración de este análisis, cómo es que estos organismos determinaron su criterios de evaluación y la influencia de estos en la calidad académica de las IESP. Por eso es necesario que exista, para el sector de educación superior privado mexicano, una serie de estudios que establezcan los criterios básicos y convenientes con los que deben cumplir las IESP de calidad.

\section{Notas finales}

Si bien la certificación a la calidad de las IESP por parte de los tres organismos revisados representa un esfuerzo importante, la influencia real que tiene sobre el sector privado y el mejoramiento de la calidad es mínima. En razón de que son pocas las instituciones que están interesadas en certificar su calidad académica y continuará la presencia de establecimientos que no cumplen con los requisitos indispensables para ofrecer formación profesional. Independientemente de la diferencia de metodologías que ofrecen las tres organizaciones de certificación, el que una IESP se someta a dicho proceso conlleva una mejor organización de aspectos administrativos, un esfuerzo por cumplir 
los estándares importantes como poseer las instalaciones apropiadas, planta de profesores, y establecer procesos y procedimientos de evaluación (Ríos, 2001). Evidentemente, esto tiene un impacto en la formación profesional que recibe el estudiante de tales instituciones.

Ahora bien, como se constató muchas de las instituciones privadas que cuenta con las tres certificaciones son de alto perfil, porque tienen los recursos financieros y académicos para lograrlo. Esto lleva a generar la siguiente pregunta ¿qué pasa con las IESP de perfil medio y bajo que no se someten a un proceso de certificación? Es necesario reiterar que, a causa de la poca inversión destinada a la educación superior pública, los estudiantes que no pueden ingresar a la misma y que no tienen recursos económicos suficientes para pagar las altas ven en las instituciones privadas de bajo perfil y perfil medio su opción para contar con un título universitario, pero la calidad de las mismas es un aspecto que no se ha cuidado. En México no hay evaluaciones y certificaciones obligatorias que permitan conocer y difundir el estado de la calidad de las instituciones privadas; como se ha visto, solo las IESP de alto perfil se interesan por certificar su calidad académica.

Uno de los aspectos que destaca de la política educativa es garantizar cobertura de educación pero con calidad. Al satisfacer la demanda de educación superior mediante las IESP de perfil medio y perfil bajo, es decir, instituciones con una calidad académica desconocida, se deja de lado un aspecto esencial: la equidad en educación. Esto implica que solamente quienes tienen mayores recursos económicos pueden aspirar a una educación superior de calidad y los estudiantes con menores ingresos reciben una formación profesional que, a ojos de los empleadores y la sociedad, puede ser dudosa. No se puede suponer que por pagar altas colegiaturas se accede a una educación de calidad y viceversa; ya que el dinero no es una garantía o parámetro de calidad. Si el Estado mexicano ha decidido que para garantizar la cobertura de educación superior se requiere de instituciones privadas, entonces se deben establecer mecanismos que aseguren que un estudiante al elegir cualquier institución privada va a contar con la formación adecuada, los profesores calificados y las instalaciones necesarias. De no ser así se dará pie a falsas suposiciones sobre si determinadas instituciones cumplen o no con la calidad 
académica y se dará lugar a valoraciones acerca de que si determinados títulos profesionales tienen más valor que otros. Esto afectará a los egresados de las instituciones privadas de perfil bajo y medio, ya que en el mercado de trabajo es posible que sus estudios profesionales tengan una valoración negativa de parte de los empleadores. Ante esto, es preciso insistir que México necesita de programas obligatorios para la evaluación y certificación de calidad del sector de educación superior privado. Ello requiere del establecimiento de un proceso de evaluación homogéneo que valore las condiciones indispensables con las que deben operar las IESP. Con ello se garantizará la equidad (no solo de cobertura del nivel, sino de calidad) para los estudiantes que asisten a las diferentes instituciones privadas.

\section{Referencias bibliográficas}

Acosta, A. (2005) La educación superior privada en México, Digital Observatory for Higher Education in Latin America and the Caribbean, Venezuela, Instituto Internacional de la UNESCO para la Educación Superior en América Latina y el Caribe, Organización de las Naciones Unidas para la Educación, la Ciencia y la Cultura.

ANUIES (2010) "La ANUIES", Asociación Nacional de Universidades e Institutos de Educación Superior. Obtenida el 30 de septiembre de 2010, de <http://www.anuies.mx/la_anuies/que_es/laanuies.php>

ANUIES (2008) Anuario estadístico. Población escolar y personal docente en la educación media y superior, Asociación Nacional de Universidades e Institutos de Educación Superior, México.

Casanueva, C. (2005) Crecimiento de la educación superior privada en México: un debate inconcluso, en Teresina Bertussi (coord.), Anuario educativo mexicano: visión retrospectiva, México, Universidad Pedagógica Nacional/Miguel Ángel Porrúa, pp. 401-429.

COPAES (2010a) ¿Qué es el COPAES?: misión, Consejo para la Acreditación de la Educación Superior. Obtenida el 29 de septiembre de 2010, de $<$ http://www.copaes.org.mx/home/Antecedentes.php>

COPAES (2010b) Marco general para los procesos de acreditación de programas Académicos de nivel superior, Consejo para la Acreditación de la Educación Superior, México. Obtenida el 29 de septiembre de 2010, de <http:// www.copaes.org.mx/home/docs/docs_acred/3_Marco_general.pdfs

FIMPES (2010) Criterios de acreditación, Federación de Instituciones Mexicanas Particulares de Educación Superior, México. Obtenida 
el 23 de septiembre de 2010, de http://www.fimpes.org.mx/index. php?option=com_content $\&$ view=article $\&$ id $=14 \&$ Itemid $=21$

FIMPES (2009) Manual para la aplicación del sistema de acreditación, Federación de Instituciones Mexicanas Particulares de Educación Superior, México. Obtenida el 23 de septiembre de 2010, de <http://www.fimpes.org. mx/phocadownload/015_manual-fimpes.pdf\#page=9>

Fresán, M. y Taborga H. (2002) Indicadores y parámetros para el ingreso y la permanencia de instituciones de educación superior a la ANUIES, México.

Gil-Antón, M. (2008) Los académicos en instituciones privadas. Una aproximación a otros actores, hoy en la sombra, en Revista de la Educación Superior, vol. XXXVII, n. ${ }^{\circ}$ 145, pp. 115-121, México, Asociación Nacional de Universidades e Institutos de Educación Superior.

De Leonardo, P. (1983) La educación superior privada en México. Bosquejo histórico, Línea, México.

Levy, D. (1995) La educación superior y el Estado en Latinoamérica. Desafíos privados al predominio público, Centro de Estudios sobre la Universidad, Universidad Nacional Autónoma de México, México, Miguel Ángel Porrúa.

Mendoza, J. (2004) La educación superior privada, en Pablo Latapí (coord.), Un siglo de educación en México (tomo 2), pp. 325-354, Fondo de Cultura Económica, México.

Muñoz I., C. (1981) Socioeconomía de la educación privada y pública. El caso de México, en Revista Latinoamericana de Estudios Educativos, vol. XI, n. ${ }^{\circ}$ 1, pp. 111-132, México.

Olivier, G. (2005) Educación superior privada en México. Veinte años de expansión: 1982-2002, tesis de maestría en Pedagogía, Facultad de Filosofía y Letras, Universidad Nacional Autónoma de México, México.

Ríos, C. (2001) Procesos de acreditación y evaluación en los EE.UU. y México: Un estudio comparativo, Revista de la Educación Superior, n. ${ }^{\circ}$ 119, pp. 1-9, ANUIES, México. Obtenida el 15 de noviembre de 2010, de <http:// www.anuies.mx/servicios/p_anuies/publicaciones/revsup/res119/art5. htm>

SEP (2002) Acuerdo n. ${ }^{\circ} 279$ por el que se establecen los trámites y procedimientos relacionados con el Reconocimiento de Validez Oficial de Estudios de Tipo Superior, Secretaría de Educación Pública, publicado en Diario Oficial de la Federación el 10 de julio de 2000. Obtenido el 30 de abril 2010, de <http://www.univalletlax.edu.mx/include/Acuerdo\%20No.\%20 $279 \% 20($ SEP).pdf $>$ 
Silas, J. C. (2005) Realidades y tendencias en la educación superior privada mexicana, en Perfiles Educativos, vol. XXVII, n. ${ }^{\circ} 109-110$, pp. 7-3, México, Centro de Estudios sobre la Universidad, Universidad Nacional Autónoma de México.

Subsecretaría de Educación Superior (2010) Consulta de programas educativos de calidad. Evaluación y acreditación en cifras. Obtenida el 23 de septiembre de 2010, de <http://ses.sep.gob.mx/wb/ses/evaluacion_y_calidad>

Vega-Tanto, G. (2009) Poniendo orden a las instituciones particulares de educación superior en México: una taxonomía aplicada a su complejidad y diversidad, en Revista de la Educación Superior, vol. XXXVIII, n. ${ }^{\circ}$ 150, pp. 37-60. Asociación Nacional de Universidades e Institutos de Educación Superior, México.

Villaseñor, G. (2004) La función social de la educación superior en México. La que es y la que queremos que sea, Universidad Autónoma Metropolitana/ Universidad Veracruzana/ Centro de Estudios sobre la Universidad, Universidad Nacional Autónoma de México, México.

\section{Índice de siglas}

ANUIES: Asociación Nacional de Universidades e Institutos de Educación Superior.

CONACyT: Consejo Mexicano de Ciencia y Tecnología.

COPAES: Consejo para la Acreditación de la Educación Superior.

FIMPES: Federación de Instituciones Mexicanas Particulares de Educación Superior.

IESP: Instituciones de Educación Superior Privadas.

ITAM: Instituto Tecnológico Autónomo de México.

ITESM: Instituto Tecnológico de Estudios Superiores de Monterrey.

ITESO: Instituto Tecnológico y de Estudios Superiores de Occidente.

RVOE: Reconocimiento de Validez Oficial de Estudios.

SEP: Secretaría de Educación Pública.

UIA: Universidad Iberoamericana.

ULSA: Universidad Lasalle.

Recibido: 1/03/2011

Aceptado: 27/06/ 2011 